Schmerz 2011 $\cdot 25: 440-443$

DOI 10.1007/s00482-011-1049-9

(c) Deutsche Gesellschaft zum Studium des Schmerzes. Published by Springer-Verlag all rights reserved 2011
Originalartikel

Krummenacher $\mathrm{P}$, Candia V, Folkers $\mathrm{G}$ et al (2010) Prefrontal cortex modulates placebo analgesia. Pain 148(3):368-374

\section{Hintergrund}

Die Placebo-Analgesie gilt als eines der eindrücklichsten Beispiele für den Placeboeffekt. Diese zeigt, wie höhere Hirnfunktionen, z. B. die Erwartungshaltung bzw. Überzeugung von Patienten und/ oder gesunden Probanden bezüglich der Wirksamkeit einer therapeutischen Intervention, die Schmerzwahrnehmung signifikant modulieren können $[1,2]$.

Der dorsolaterale präfrontale Kortex (DLPFC) wurde vielfach mit kognitiven sog. „Top-down“-Prozessen der Schmerzregulation $[1,3]$ sowie Aspekten der Entstehung, Aufrecherhaltung und Manipulation von kognitiven Repräsentationen in Verbindung gebracht [4]. In unserer experimentellen Studie mit 40 gesunden, rechtshändigen Männern haben wir mithilfe der interventionellen Methode der nichtinvasiven, repetitiven transkraniellen Magnetstimulation (rTMS) die Rolle des rechten und linken präfrontalen Kortex (PFC) bei der Vermittlung von erwartungsbedingten Placebo-Analgesie-Effekten in einem Hitzeschmerzreiz-Paradigma analysiert. Dabei wurde das TMS-Gerät selbst auch als Placebo eingesetzt.

\section{Studiendesign}

In einer initialen multisensorischen Erwartungs-Induktionsprozedur wur-

\author{
P. Krummenacher \\ Collegium Helveticum, ETH Zürich \& Universität Zürich
}

\title{
Präfrontale Kontrolle von Erwartungen bei der Placebo-Analgesie
}

de den Probanden ein Zusammenhang zwischen vermeintlicher TMS-Intensität und verminderter Schmerzwahrnehmung suggeriert (Analgesie-Erwartung). Danach erhielten jeweils die Hälfte der Teilnehmer in der Analgesie-Erwartungs- (Suggestion: TMS = hochwirksamer Schmerzkiller) und der falschen Erwartungs-Kondition (Suggestion: TMS = Messinstrument) während 15 min entweder eine echte oder eine vorgetäuschte (Schein-TMS) repetitive 1-Hz-rTMS(MagPro X1oo; Medtronic-Dantec ${ }^{\oplus}$, Dantec Dynamics Ltd., Royal Portbury, UK) Behandlung über dem rechten und linken DLPFC in randomisierter, ausbalancierter Reihenfolge (• Abb. 1). Die Methode der TMS ermöglicht, nach dem physikalischen Prinzip der elektromagnetischen Induktion, die kortikale Erregbarkeit kurzfristig und vorübergehend zu verändern [5]. Dadurch kann hypothesengeleitet die kritische Bedeutung eines bestimmten kortikalen Netzwerks bei der Vermittlung einer höheren Hirnfunktion überprüft werden. Die Stimulationsorte über dem PFC wurden jeweils mithilfe des internationalen 102o Elektroenzephalographie (EEG)-Referenzsystems bestimmt [6].

Schmerzschwelle und -toleranz wurden jeweils an beiden Unterarmen mit einem 30×30-mm-Peltier-Element (TSAII; Medoc Ltd. Advanced Medical Systems, Ramat Yishai, Israel) zu Beginn des Experimentes (Ausgangswert) und dann nach den Schein- bzw. den echten TMSBehandlungen gemessen (• Abb. 1). Die emotionale Stimmung der Teilnehmer wurde jeweils mittels Fragebogen (mehrdimensionaler Befindlichkeitsfragebogen, MDBF, [7]) vor und unmittelbar nach jeder Stimulation erhoben (- Abb. 1). Nach der wahrgenommenen Wirksamkeit der Behandlung auf das Schmerzerlebnis wurde am Ende der letzten Schmerzmessung gefragt. Die Analgesie-Erwartung wurde von den Probanden jeweils zu Beginn und am Ende des Experimentes eingeschätzt.

\section{Ergebnisse}

Alter, Ausbildung, Ausprägungsstärke der Händigkeit und Stimmung waren in den vier Gruppen vergleichbar. Die AnalgesieErwartungen waren in beiden AnalgesieErwartungs-Gruppen ähnlich hoch ausgeprägt. Die Stimmung wurde durch die Stimulation nicht beeinflusst.

In $-\mathrm{Abb} .2$ sind die Differenzwerte (Behandlung - Ausgangswert) für die Schmerzschwelle ( $\bullet$ Abb. 2a), die Schmerztoleranz (• Abb. 2 b) sowie für die retrospektiv wahrgenommene Schmerzreduktion (• Abb. 2 c) in ${ }^{\circ} \mathrm{C}$ dargestellt. Die Werte wurden über die Stimulationsseite (rechter und linker PFC) und die gemessene Unterarmseite gemittelt.

Die Ergebnisse in - Abb. 2 zeigen, dass das nichtpharmakologische Placebo (Schein-TMS) sowohl die Schmerzschwelle (• Abb. 2a) als auch die Schmerztoleranz (• Abb. 2b) am linken und rechten Unterarm signifikant erhöht. Dieses Muster zeigt sich auch in der retrospektiv wahrgenommenen Schmerzreduktion (• Abb. 2c). Während die vorübergehende funktionelle Läsion des rech- 
ten und linken DLPFC durch rTMS (echte TMS) die Placebo-Analgesie komplett aufheben konnte, wurde die Schmerzverarbeitung per se durch die echte Stimulation über dem PFC nicht beeinflusst. Ein Ausfall der präfrontalen Kontrolle geht einher mit einem Verlust der Placeboreaktion [9].

\section{Diskussion}

Diese Befunde ergänzen bisherige klinische Erkenntnisse von Patienten mit Frontalhirndegeneration [10] sowie zahlreiche Befunde aus korrelativen pharmakologischen [11, 12, 13] und bildgebenden Verfahren $[1,14]$ und dokumentieren, dass erwartungsinduzierte Placebo-Analgesie ein aktiver neurobiologischer Prozess ist $[15,16,17]$, der von präfrontalen Netzwerken vermittelt wird. Es ist anzunehmen, dass dabei körpereigene absteigende Schmerzkontrollsysteme aktiviert werden, die zumindest partiell über die Ausschüttung endogener Opiate vermittelt werden [18].

\section{Kommentar}

Dieses neue interventionelle TMS-Setting könnte möglicherweise dazu beitragen, besser zwischen assoziativen Lernund Erwartungsmechanismen bei Placebo-Analgesieprozessen im Rahmen der neurokognitiven Schmerzforschung [19] differenzieren zu können.

\section{Maximierung des psychosozialen Kontexts in der praktischen Medizin}

Ein besseres Verständnis der neuropsychopharmakologischen Mechanismen von Placeboeffekten trägt dazu bei, die Effekte einer medizinischen Behandlung gezielt, kontextabhängig und individuell durch psychologische Interventionen $\mathrm{zu}$ unterstützen und zu maximieren. Dies könnte durch die Befragung von bisherigen Erfahrungen, Einstellungen und Überzeugungen, in Kombination mit beratenden Aufklärungsgesprächen, realisiert werden.

Denkbar wäre auch die Entwicklung von neuen, ethisch vertretbaren Stimulationsprotokollen mit dem Ziel, negati-

Schmerz 2011 - 25:440-443 DOI 10.1007/s00482-011-1049-9

(c) Deutsche Gesellschaft zum Studium des Schmerzes.

Published by Springer-Verlag - all rights reserved 2011

\section{P. Krummenacher}

\section{Präfrontale Kontrolle von Erwartungen bei der Placebo-Analgesie}

\section{Zusammenfassung}

Erwartungen und Überzeugungen modulieren das Schmerzempfinden. Dies wird besonders deutlich bei der Placebo-Analgesie. Der dorsolaterale präfrontale Kortex (DLPFC) wird mit Prozessen der Schmerzregulation sowie mit Aspekten der Erzeugung, Aufrechterhaltung und Manipulation von kognitiven Repräsentationen in Verbindung gebracht. In einem Hitzeschmerzreiz-Paradigma wurde eine nichtinvasive niederfrequente repetitive transkranielle Magnetstimulation (rTMS) eingesetzt, um vorübergehend die Funktion des rechten und linken DLPFC zu stören oder um das rTMS-Gerät selbst als Placebo einzusetzen, bevor eine erwartungsinduzierte Placebo-Analgesie angewendet wur- de. Die Ergebnisse zeigen, dass die Scheinbehandlung signifikant Schmerzschwelle und -toleranz erhöhen konnte. Während die rTMS das Schmerzempfinden nicht beeinträchtigte, hob sie doch vollständig die Placebo-Analgesie auf. Diese Ergebnisse lassen vermuten, dass die erwartungsinduzierte Placebo-Analgesie vom präfrontalen Kortex beider Hemisphären vermittelt wird. Mögliche Implikationen für die praktische Medizin und klinische Forschung werden im Artikel diskutiert.

\section{Schlüsselwörter}

Placebo-Analgesie · Präfrontaler Kortex · Schmerz $\cdot$ Erwartung $\cdot$ Transkranielle Magnetstimulation

\section{Prefrontal cortex mediated control of expectations in placebo analgesia}

\section{Abstract}

Expectations and beliefs modulate the experience of pain, which is particularly evident in placebo analgesia. The dorsolateral prefrontal cortex (DLPFC) has been associated with pain regulation and with the generation, maintenance and manipulation of cognitive representations. In a heat-pain paradigm, we employed non-invasive low-frequency repetitive transcranial magnetic stimulation (rTMS) to transiently disrupt left and right DLPFC function or used the TMS device itself as a placebo, before applying an expectation-induced placebo analgesia. The results demonstrated that placebo significantly increased pain threshold and pain tolerance. While rTMS did not affect pain experience, it completely blocked placebo analgesia. These findings suggest that expectation-induced placebo analgesia is mediated by symmetric prefrontal cortex function. Possible implications for medical practice and clinical trial research will be discussed in the article.

\section{Keywords}

Placebo analgesia · Prefrontal cortex · Pain . Expectation - Transcranial magnetic stimulation 


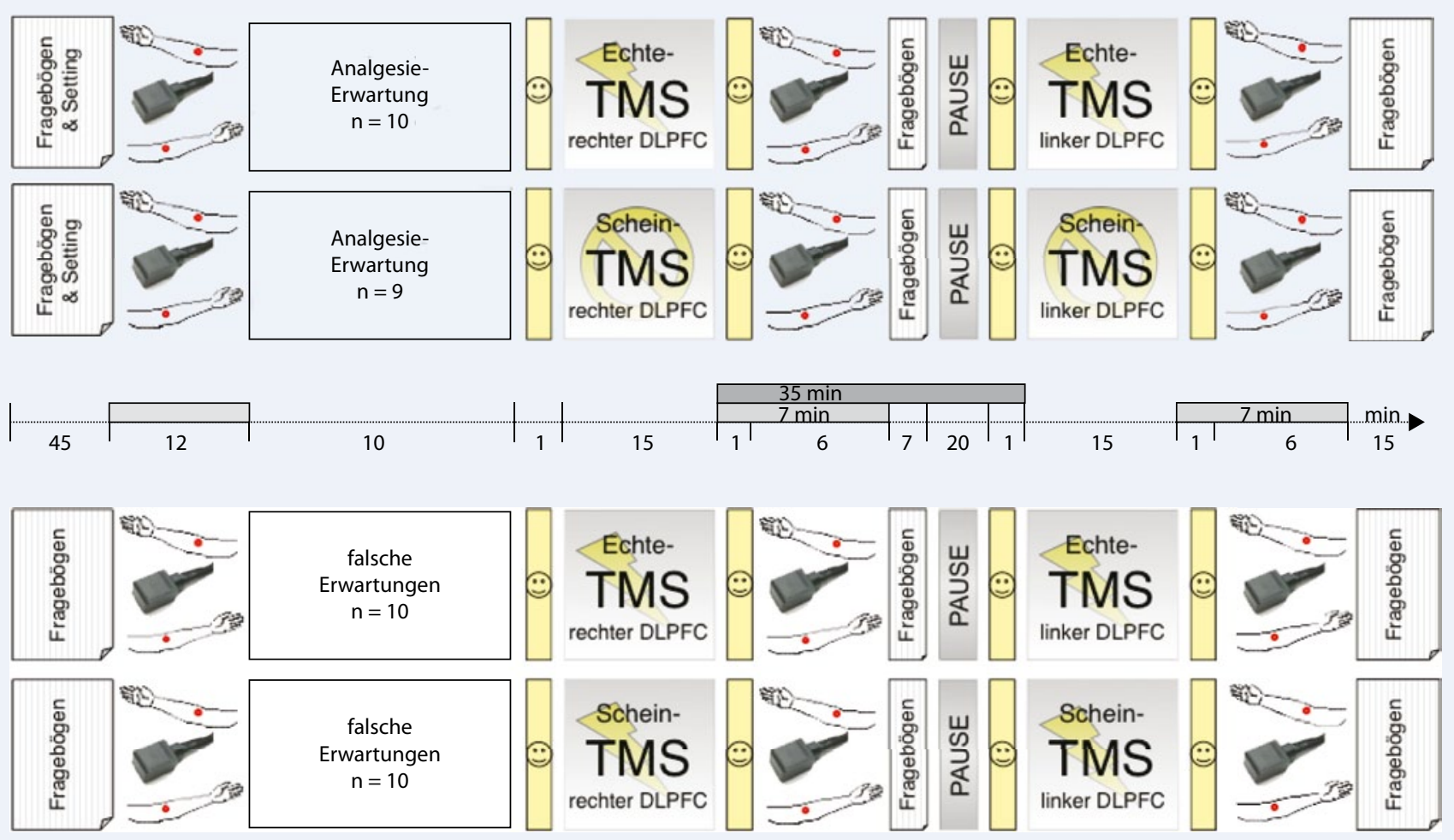

Abb. $1 \Delta$ Experimentelles Design. Nach einer initialen multisensorischen Erwartungs-Induktionsprozedur erhielten jeweils die Hälfte der Teilnehmer in der Analgesie-Erwartungs- (Suggestion:TMS = hochwirksamer Schmerzkiller) und der falschen Erwartungs-Kondition (Suggestion:TMS = Messinstrument) während 15 min entweder eine echte oder eine vorgetäuschte (Schein-TMS) repetitive 1-Hz-rTMS-Behandlung über dem rechten und linken DLPFC in randomisierter, ausbalancierter Reihenfolge. Schmerzschwelle und -toleranz wurden jeweils an beiden Unterarmen zu Beginn des Experimentes (Ausgangswert) und dann nach den Schein- bzw. den echten TMS-Behandlungen gemessen. TMS transkranielle Magnetstimulation; rTMS repetitive transkranielle Magnetstimulation; DLPFC dorsolateraler präfrontaler Kortex. (Mit freundl. Genehmigung von PAIN $^{\circledR}$, IASP$^{\circledR}$, aus [8])

ve Erwartungen zu hemmen (sog. Noceboeffekte) und positive Erwartungen zu verstärken. Aus Sicherheitsgründen haben wir nur ein Niederfrequenz-Stimulationsprotokoll verwendet. Dieser Forschungsansatz könnte aber in Zukunft mittels neuen Stimulationsprotokollen, wie z. B.

1. Hochfrequenz-TMS,

2. Theta-Burst-Stimulation (TBS) oder

3. transkranieller Gleichstromstimulation

weiterentwickelt werden. Erfahrungswerte zeigen, dass solche Protokolle die kortikale Erregbarkeit erleichtern. Vielversprechend wäre es auch, zu erforschen, ob durch neue Arten von Stimulationsprotokollen längerfristige Effekte erzielt werden könnten, die dann z. B. bei Patienten mit chronischen Schmerzen eingesetzt werden könnten.

\section{Minimierung des} psychosozialen Kontexts in der klinischen Forschung

Die Analyse von neuropsychologischen Wirkmechanismen von Placeboeffekten ist auch für die Entwicklung von neuen pharmakologischen Substanzen bedeutsam. Wenn sich Placeboeffekte in klinischen Studien kontrollieren und minimieren lassen, kann der Wirkungsgrad einer neu entwickelten pharmakologischen Substanz oder einer anderen medizinischen Behandlung wesentlich genauer bestimmt werden [20]. In diesem Zusammenhang kann der mögliche Einsatz unseres TMS-Paradigmas - als Ergänzung zum Offen-verdeckt-Paradigma [21] - in klinischen Studien diskutiert werden, indem der unerwünschte, konfundierende erwartungsabhängige Placeboeffekt kurzfristig ausgeschaltet werden könnte.

\section{Limitationen}

Die neuropsychologische Untersuchung der Placebo-Analgesie mittels TMS erfordert die Fragmentierung eines komplexen mentalen Prozesses und seiner zugrundeliegenden neuronalen Netzwerke in einzelne Komponenten. In Folgestudien gilt es, nicht nur die interindividuelle Neuroanatomie besser zu berücksichtigen, z. B. durch Magnetresonanztomographie-gestützte Echtzeit-Neuronavigation, sondern auch die Spezifität des DLPFC bei der erwartungsbedingten Placebo-Analgesie durch den Einsatz von weiteren kortikalen Kontrollregionen sowie deren Konnektivität zu untersuchen. Dadurch könnten sich möglicherweise auch neue Erkenntnisse im Hinblick auf die in unserer Studie nicht gefundene unterschiedliche Beteiligung der beiden Hemisphären bei der Vermittlung von Placeboeffekten ergeben. 


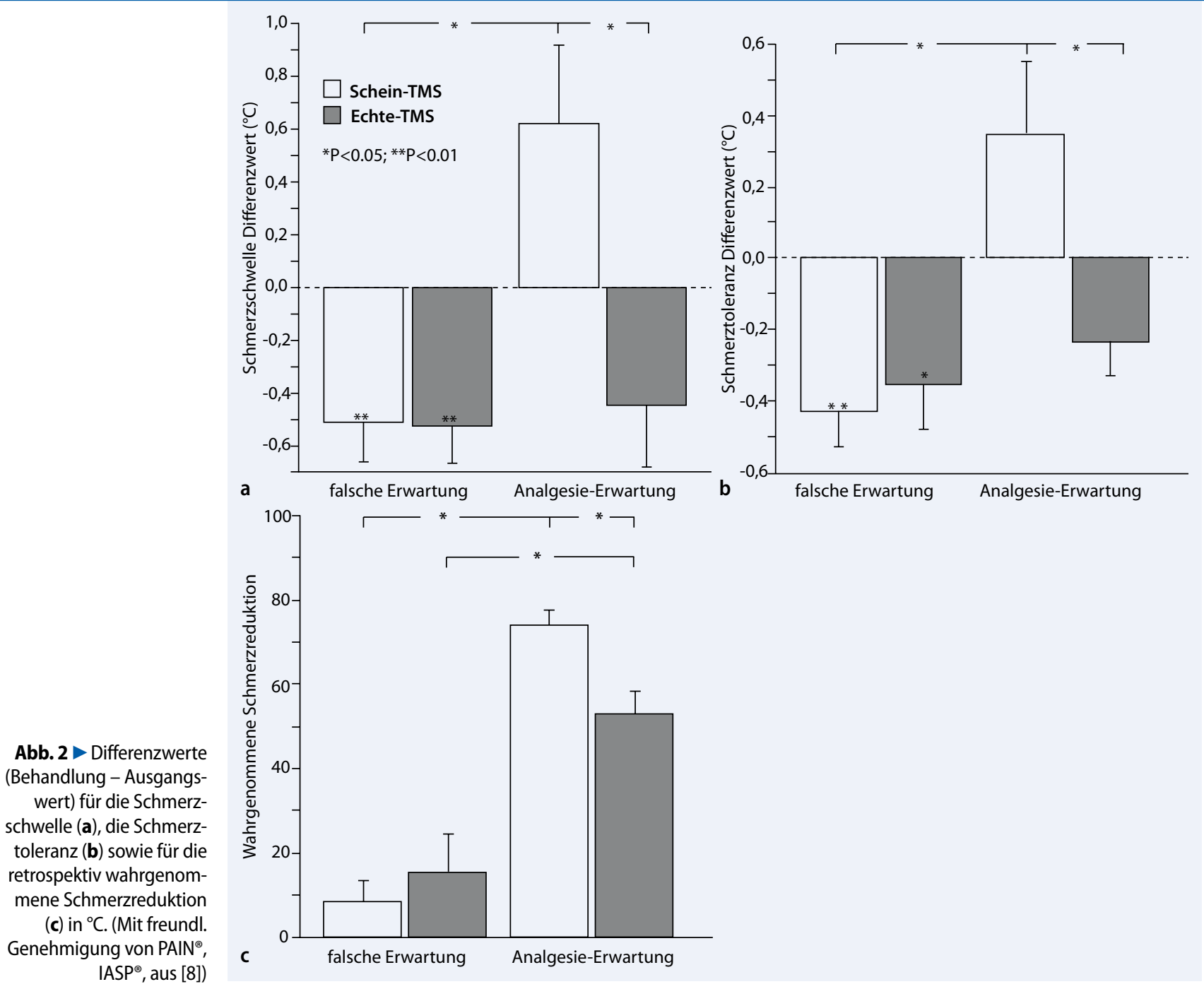

\section{Korrespondenzadresse}

\section{Dr. P. Krummenacher}

Collegium Helveticum,

ETH Zürich \& Universität Zürich

Schmelzbergstr. 25, 8092 Zürich

Schweiz

krummenacher@collegium.ethz.ch

Interessenkonflikt. Der korrespondierende Autor gibt an, dass kein Interessenkonflikt besteht.

\section{Literatur}

1. Wager TD et al (2004) Placebo-induced changes in FMRI in the anticipation and experience of pain. Science (New York, N.Y.) 303(5661):1162-1167

2. Kong J et al (2006) Brain activity associated with expectancy-enhanced placebo analgesia as measured by functional magnetic resonance imaging. J Neurosci 26:381-388

3. Basbaum Al, Fields HL (1984) Endogenous pain control systems: brainstem spinal pathways and endorphin circuitry. Annu Rev Neurosci 7:309-338

4. Miller EK, Cohen JD (2001) An integrative theory of prefrontal cortex function. Annu Rev Neurosci 24:167-202
5. Ridding MC, Rothwell JC (2007) Is there a future for therapeutic use of transcranial magnetic stimulation? Nature reviews 8:559-567

6. Herwig U, Satrapi P, Schonfeldt-Lecuona C (2003) Using the international 10-20 EEG system for positioning of transcranial magnetic stimulation. Brain Topogr 16:95-99

7. Steyer R, Schwenkmezger P, Notz P, Eid M (1997) MDBF - Mehrdimensionaler Befindlichkeitsfragebogen, Hogrefe, Göttingen, Deutschland

8. Krummenacher P, Candia V, Folkers G et al (2010) Prefrontal cortex modulates placebo analgesia. Pain 148:368-374

9. Benedetti $F$ (2010) No prefrontal control, no placebo response. Pain 148:357-358

10. Benedetti F et al (2006) Loss of expectation-related mechanisms in Alzheimer's disease makes analgesic therapies less effective. Pain 121:133-144

11. Eippert F et al (2009) Activation of the opioidergic descending pain control system underlies placebo analgesia. Neuron 63:533-543

12. Amanzio M, Benedetti F (1999) Neuropharmacological dissection of placebo analgesia: expectationactivated opioid systems versus conditioning-activated specific subsystems. J Neurosci 19:484-494

13. Levine JD, Gordon NC, Fields HL (1978) The mechanism of placebo analgesia. Lancet 2(8091):654657
14. Petrovic $P$, Kalso E, Petersson KM, Ingvar M (2002) Placebo and opioid analgesia - imaging a shared neuronal network. Science 295(5560):1737-1740

15. Enck P, Benedetti F, Schedlowski M (2008) New insights into the placebo and nocebo responses. Neuron 59:195-206

16. Benedetti F, Carlino E, Pollo A (2011) How placebos change the patient's brain. Neuropsychopharmacology 36:339-354

17. Finniss DG, KaptchukTJ, Miller F, Benedetti F (2010) Biological, clinical, and ethical advances of placebo effects. Lancet 375(9715):686-695

18. Bingel U (2010) Mechanisms of endogenous pain modulation illustrated by placebo analgesia: functional imaging findings. Schmerz 24:122-129

19. Wiech K, Ploner M, Tracey I (2008) Neurocognitive aspects of pain perception. Trends Cogn Sci 12:306-313

20. Schedlowski M, Rief W, Enck P (2010) Placebo- und Noceboeffekte: Die große Herausforderung für die Verhaltenswissenschaften in der Medizin. Z Med Psychol 19:1-3

21. Colloca L, Lopiano L, Lanotte M, Benedetti F (2004) Overt versus covert treatment for pain, anxiety, and Parkinson's disease. Lancet Neurol 3:679-684 